\title{
Linear pneumatic motors - a comparative study
}

\author{
Tudor Deaconescu*, and Andrea Deaconescu
}

Transilvania University of Braşov, Department of Industrial Engineering and Management, 500036, 29 Bd. Eroilor, Braşov, Romania

\begin{abstract}
The paper presents a comparative study of the performance of single-acting cylinders, diaphragm cylinders and pneumatic muscles, and offers users information that allows the selection of an optimum technical solution. Such a study was necessary, in view of the numerous papers on pneumatic muscle applications found in literature, that assert the superiority of pneumatic muscles over other pneumatic linear motors in relation to quantities like dimensions, mass, developed force or energy-to-mass ratios, however without offering concrete data.
\end{abstract}

\section{Introduction}

The increasingly higher requirements related to the performance parameters of manufacturing systems fuel the competition between the various actuation modalities: mechanical, electrical, or fluid. This generates the continued improvement of each type of actuation and their convergence towards performance maximisation.

Compressed air is one of the most efficient means of actuation and automation of manufacturing systems. Over time pneumatic actuations have undergone continuous development, with a gradual passing form the utilisation of individual components to the deployment of complex automation systems. New systems were conceived, adding mechanical, electronic and sensor elements to the classical pneumatic structures.

The increasingly larger scale of compressed air utilisation in industrial applications is due to its benefits, like easy generation and storage, non-flammability, minimum explosion risk, the minimal maintenance effort required by pneumatic systems, etc.

The linear pneumatic motors used in industry are rod or rodless cylinders, diaphragm cylinders, bellows cylinders, as well as pneumatic muscles.

Further on the paper presents a comparative study of the performance of rod cylinders, diaphragm cylinders and pneumatic muscles, offering users the necessary information for selecting the optimum solution for each application. For the same size of the three types of pneumatic motors (diameter, stroke) the comparison refers to dimensions, masses, developed forces and energy-to-mass ratios.

Such a study was called for, in view of the numerous papers on pneumatic muscle applications found in literature, that assert the superiority of these parameters in pneumatic muscles over other pneumatic linear motors, however without offering concrete data [1-3].

\footnotetext{
*Corresponding author: tdeacon@unitbv.ro
} 


\section{The analysed pneumatic motors}

The aim of the study discussed in this paper is to highlight the performance of pneumatic muscles, as compared to linear motors whose developed forces are not constant over the entire stroke length. These are single-acting cylinders and diaphragm cylinders.

Single-acting cylinders are used when force needs to be applied in a single direction, typically for feed motions. The return of the piston is automatic, by means of a built-in spring. The force developed by such a cylinder is [4]:

$$
F=p \cdot \frac{\pi \cdot D^{2}}{4}-F_{F R}-F_{S P R}
$$

where $p$ is the pressure of the compressed air, $D-$ the piston diameter, $F_{F R}-$ the friction forces, $F_{S P R}$ - the force of the spring.

Diaphragm cylinders are used in gripping devices and transfer arms of industrial robots, clamping devices of machine tools, stopper devices, etc. [4]. By eliminating the conventional sliding piston seal, diaphragm cylinders produce nearly frictionless motion, except for the miniscule amount of friction at the rod seal [5]. The force developed by these cylinders is determined by equation (2) [4]:

$$
F=S_{e} \cdot p-F_{S P R}
$$

where $S_{e}$ denotes the effective diaphragm area.

The pneumatic muscle is a motor that causes displacement by modifying its geometrical form under pressure. As compressed air is fed into the muscle a traction force is generated along its longitudinal axis, and the muscle shortens in proportion with the increase in internal pressure. The pneumatic muscle reaches the maximum developed force as soon as contraction starts, which force decreases to zero by the end of the stroke. The force developed by a pneumatic muscle is given by equation (3) [6]:

$$
F=p \cdot \frac{\pi}{4} \cdot d^{2} \cdot\left[\frac{3 \cdot \cos ^{2} \alpha-1}{1-\cos ^{2} \alpha}\right]
$$

where $p$ is the working pressure, $d$ the interior diameter of the pneumatic muscle, and $\alpha$ is the wrapping angle of the synthetic fibre mesh that envelopes the interior tube.

From the three categories of actuators described above, the following models were selected for the comparative study of linear pneumatic motor performance:

- $\quad$ a single-acting cylinder model ESNU ISO 6432, manufactured by FESTO, Germany;

- a diaphragm cylinder model MM-1 (349-180-007) manufactured by TECO Pneumatic, USA;

- a pneumatic muscle, model MAS-20-N, manufactured by FESTO Germany [7].

In the single-acting cylinder and the pneumatic muscle the piston and tube diameter, respectively, is of $20 \mathrm{~mm}$, while the diaphragm cylinder diameter is of $17.7 \mathrm{~mm}(0.7 \mathrm{in})$. The strokes of the former two types of motors are of $25 \mathrm{~mm}$, and the stroke of the diaphragm cylinder is of $17.7 \mathrm{~mm}(0.7 \mathrm{in})$. Figure 1 shows the three discussed linear motors.

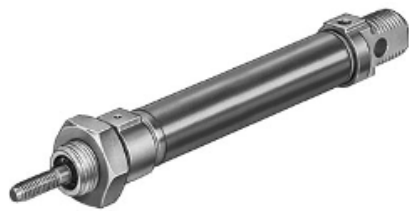

Single-acting cylinder

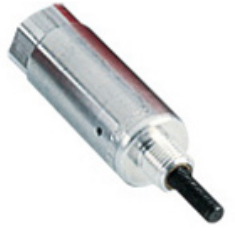

Diaphragm cylinder

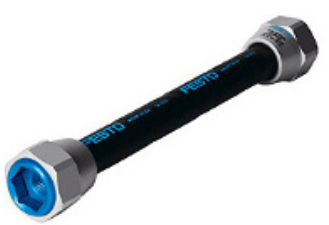

Pneumatic muscle

Fig. 1. Linear pneumatic motors considered for comparison. 


\section{Comparative studies}

The comparative analysis of the performance of linear pneumatic motors is based on a set of assessment criteria that take into consideration motor dimensions and mass, the developed forces and the energy-to-mass ratio.

From the viewpoint of overall size of the three types of motors, Table 1 centralises the main values, as indicated in the technical documentation of these devices.

Table 1. Dimensions of the analysed pneumatic motors.

\begin{tabular}{|c|c|c|}
\hline Motor type & $\begin{array}{c}\text { Total length, } \\
\text { including } \\
\text { assembly } \\
\text { elements } \\
{[\mathbf{m m}]}\end{array}$ & $\begin{array}{c}\text { Maximum } \\
\text { exterior } \\
\text { diameter } \\
\text { [mm] }\end{array}$ \\
\hline Single-acting cylinder & 132 & 36 \\
\hline Diaphragm cylinder & 71.374 & 25.4 \\
\hline Pneumatic muscle & 333 & 30 \\
\hline
\end{tabular}

It can be noticed that as to the axial dimensions the pneumatic muscle is the longest, and the diaphragm cylinder the shortest motor. As to the radial size, the three motors have comparable dimensions, with a slight advantage of the diaphragm cylinder.

The masses of the pneumatic motors depend on the lengths of their strokes. In singleacting cylinders the masses corresponding to a $0 \mathrm{~mm}$ stroke is of $0.167 \mathrm{~kg}$, and increases by $0.0072 \mathrm{~kg}$ for each $10 \mathrm{~mm}$ of stroke. Equation (4) is the linear function that describes the dependency of the pneumatic cylinder mass on the stroke $(s)[8]$ :

$$
m_{E S N U}(s)=0.0072 \cdot s+0.167
$$

In the case of the analysed cylinder whose stroke is of $25 \mathrm{~mm}$, the corresponding mass is of $0.185 \mathrm{~kg}$.

In the case of the diaphragm cylinder, its mass as indicated by the manufacturer is of $0.162 \mathrm{~kg}$.

While the mass of artificial muscles is evidently directly dependent on their length, it is smaller than that of a pneumatic cylinder of the same dimensions due to the deployed materials. Figure 2 presents the dependency of the analysed pneumatic muscle mass on its length. The graph was plotted based on the information provided by the MuscleSIM 2.0.1.5 application provided by the manufacturer.

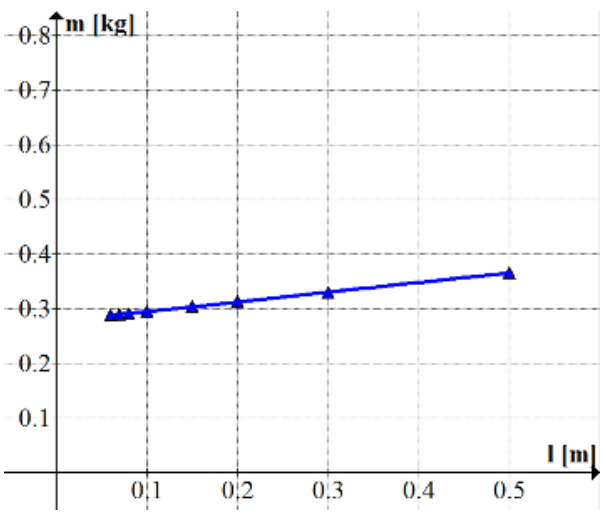

Fig. 2. Dependency of the pneumatic muscle mass on its length. 
Equation (5) represents the linear function that describes best the dependency of the pneumatic muscle mass on its length [8]:

$$
m_{20}(l)=0.178 \cdot l+0.277
$$

where $l$ denotes the length of the active part of the pneumatic muscle (length of the elastic tube). For the analysed muscle whose active length is of $162.9 \mathrm{~mm}$, the corresponding mass is of $0.306 \mathrm{~kg}$.

The determined values of the masses of the three motors reveal the pneumatic muscle as the heaviest and the diaphragm cylinder as the lightest actuator.

Table 2 presents the forces developed by the three pneumatic motors. The maximum working pressure taken into consideration was of 6 bar.

Table 2. Forces developed by the analysed pneumatic motors.

\begin{tabular}{|c|c|c|}
\hline Motor type & $\begin{array}{c}\text { Maximum force } \\
\mathbf{F}_{\max }[\mathbf{N}]\end{array}$ & $\begin{array}{c}\text { Minimum force } \\
\mathbf{F}_{\min }[\mathbf{N}]\end{array}$ \\
\hline Single-acting cylinder & 169 & 16.5 \\
\hline Diaphragm cylinder & 139.74 & 31.15 \\
\hline Pneumatic muscle & 1200 & $330(\text { at } \varepsilon=0.2)^{*}$ \\
\hline${ }^{*} \varepsilon=$ the specific axial contraction
\end{tabular}

Based on the data in Table 2, Figure 3 shows the plotted lines of the force variation versus stroke in the three analysed pneumatic motors.

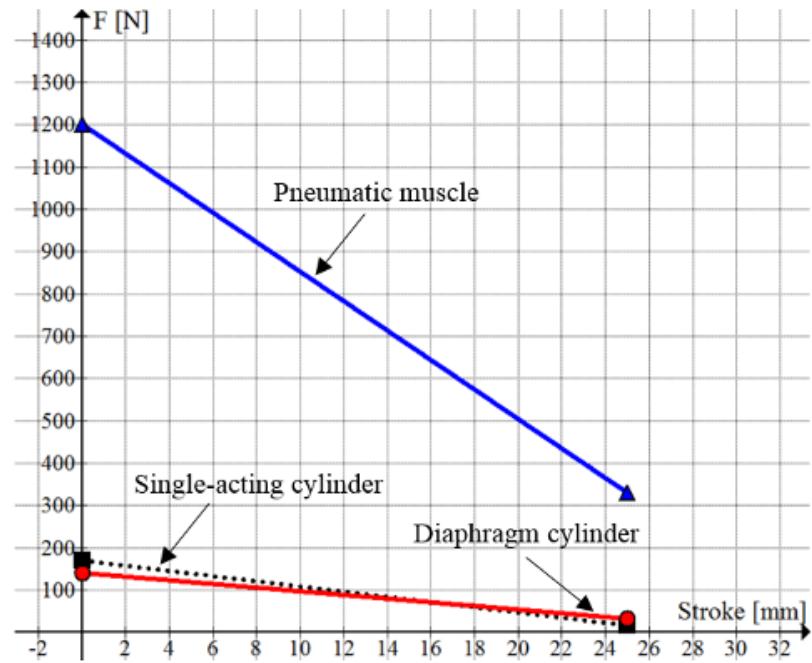

Fig. 3. Variation of the developed force versus stoke in the three linear motors.

The graph reveals clearly that the forces developed by the pneumatic muscles are 8 to 10 times greater than those generated by single-acting cylinders and diaphragm cylinders, respectively.

In the case of the pneumatic muscle however, the dependency of the force on the stroke $s$ is not linear. Equation (6) [8] represents the computational relationship of the force developed by the pneumatic muscle in dependence on the specific axial contraction $\varepsilon=s / l$.

$$
F_{20}(\varepsilon)=1.678 \cdot 10^{5} \cdot \varepsilon^{4}-1.087 \cdot 10^{5} \cdot \varepsilon^{3}+2.924 \cdot 10^{4} \cdot \varepsilon^{2}-8640.755 \cdot \varepsilon+1490.71
$$


Another criterion of comparison of linear pneumatic motors concerning their energy efficiency is the energy-to-mass ratio. Various expressions of this indicator for pneumatic muscles can be found in [8] and [9]. Thus paper [8] puts forward a computational relationship of the energy-to-mass ratio that takes into consideration the specific axial contraction $\varepsilon$ of the pneumatic muscle, its length $l$ and mass $m$.

$$
R_{\frac{E}{m}}=\frac{l \cdot \int F(\varepsilon) \cdot d \varepsilon}{m}
$$

Starting from equation (6) and based on the graph of Figure 4, equation (8) computes the energy developed by the analysed pneumatic muscle:

$$
E_{20}(l)=l \cdot \int_{0.038}^{0.25} F_{20}(\varepsilon) \cdot d \varepsilon=130.6123 \cdot l
$$

The lower limit of the integral above (0.038) represents the minimum specific axial contraction starting at which the muscle generates the force; the upper limit of the integral above $(0.25)$ is the maximum specific axial contraction at that the muscle still generates a minimal force.

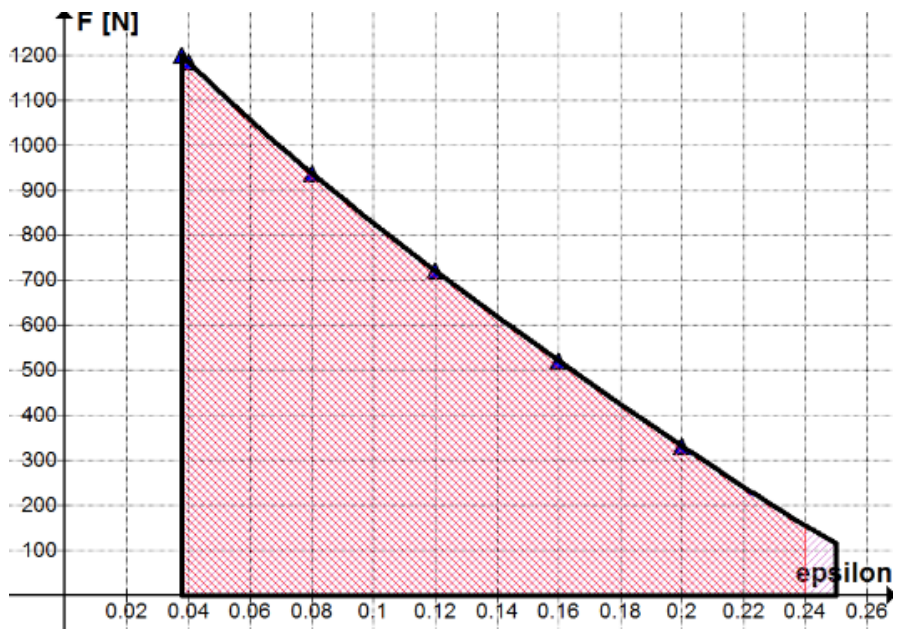

Fig. 4. Working range of the Festo MAS-20-N Fluid Muscle.

When the specific axial contraction is $0.25(\varepsilon=0.25)$, the force developed by the muscle is of about $120 \mathrm{~N}$, smaller than indicated in Table $2(\mathrm{~F}=330 \mathrm{~N}$ for $\varepsilon=0.20)$.

Equation (9) obtained by inserting equation (8) into equation (7) calculates the energyto-mass ratio of the studied pneumatic muscle:

$$
R_{\frac{E}{m}, 20}=\frac{E_{20}(l)}{m_{20}(l)}=\frac{130.6123 \cdot l}{0.306}
$$

For the two pneumatic cylinders the relationships for the energy-to-mass ratios are:

$$
\begin{aligned}
& R_{\frac{E}{m}, E S N U}=\frac{F \cdot s}{m}=\frac{(-6.1 \cdot s+169) \cdot s}{0.185} \\
& R_{\frac{E}{m}, M M-1}=\frac{F \cdot s}{m}=\frac{(-4.3436 \cdot s+139.74) \cdot s}{0.162}
\end{aligned}
$$

Based on the above equations, Table 3 features the comparison of the calculated values of the energy-to-mass ratio for the three types of analysed linear pneumatic motors. 
Table 3. Comparison of the energy-to-mass ratios.

\begin{tabular}{|c|c|}
\hline Motor type & $\begin{array}{c}\text { Energy-to-mass } \\
\text { ratio }\end{array}$ \\
\hline Single-acting cylinder & 22.82 \\
\hline Diaphragm cylinder & 15.26 \\
\hline Pneumatic muscle & 69.46 \\
\hline
\end{tabular}

The analysis of these data shows that the pneumatic muscle ensures an energy-to-mass ratio superior to other types of pneumatic motors of similar dimensions.

\section{Conclusion}

Table 4 presents a synopsis of the results yielded by the comparison of the three types of linear pneumatic motors.

Table 4. Synopsis of the results.

\begin{tabular}{|c|c|c|c|c|}
\hline Motor type & Dimensions & Masses & Forces & $\begin{array}{c}\text { Energy-to- } \\
\text { mass ratio }\end{array}$ \\
\hline Single-acting cylinder & $\odot$ & $\oplus$ & $\odot$ & $\oplus$ \\
\hline Diaphragm cylinder & $\odot$ & $\oplus$ & $\odot$ & $\oplus$ \\
\hline Pneumatic muscle & $\odot$ & $\odot$ & $\odot$ & $\oplus$ \\
\hline
\end{tabular}

An overall assessment of the results reveals that pneumatic muscles, although currently insufficiently known and applied, offer numerous benefits. In future, based on detailed knowledge of their advantages, pneumatic muscles are bound to replace single-acting cylinders or diaphragm cylinders in an increasing number of applications.

\section{References}

1. N. Tsagarakis, D.G. Caldwell, Proc. of the IEEE International Conference on Robotics \& Automation, San Francisco, USA, pp. 3641-3646 (2000).

2. N. Nakamura, M. Sekiguchi, K. Kawashima, T. Fujita, T. Kagawa, Bath Workshop on Power Transmission \& Motion Control, (2002).

3. G.A. Medrano-Cerda, C.J. Bowler, D.G. Caldwell, Proc. of the IEEE/RSJ International Conference on Intelligent Robots and Systems, Pittsburgh, USA, 378-383, (1995).

4. E. Pashkov, Y. Osinskiy, A. Chetviorkin, Electropneumatics in Manufacturing Processes (Isdatelstovo SevNTU, Sevastopol, 2004)

5. Hydraulics \& Pneumatics. Engineering Essentials: Cylinders, http://m.hydraulicspneumatics.com/200/TechZone/Cylinders/Article/False/6423/TechZ one-Cylinders?page $=2$ Accessed: 26.12.2016

6. S. Hesse, The Fluidic Muscle in Application (Blue Digest on Automation, Esslingen, 2003).

7. Festo Fluidic Muscle DMSP/MAS, https://www.festo.com/rep/en_corp/assets/pdf/info_501_en.pdf Accessed: 26.12.2016

8. T. Deaconescu, A. Deaconescu, Int. J. of Mech., Aerospace, Ind., Mechatronic and Manuf. Eng., 10(10), 1598 - 1604, (2016).

9. D.H. Plettenburg, Proc. of the 2005 IEEE 9th International Conference on Rehabilitation Robotics, Chicago, IL, USA, 545-549 (2005). 